\title{
Associations Between Corpus Callosum Damage, Clinical Disability, and Surface-Based Homologous Inter-Hemispheric Connectivity in Multiple Sclerosis
}

Andrew W. Russo ( $\sim$ awrusso@mgh.harvard.edu )

Massachusetts General Hospital https://orcid.org/0000-0002-8456-8757

Kirsten E. Stockel

Massachusetts General Hospital

Sean M. Tobyne

Massachusetts General Hospital

Chanon Ngamsombat

Athinoula A Martinos Center for Biomedical Imaging

Kristina Brewer

Massachusetts General Hospital

\section{Aapo Nummenmaa}

Athinoula A Martinos Center for Biomedical Imaging

Susie Y. Huang

Athinoula A Martinos Center for Biomedical Imaging

Eric C. Klawiter

Athinoula A Martinos Center for Biomedical Imaging

\section{Research Article}

Keywords: inter-hemispheric functional connectivity, resting state, multiple sclerosis, corpus callosum, clinical

Posted Date: October 21st, 2021

DOI: https://doi.org/10.21203/rs.3.rs-935532/v1

License: (c) (i) This work is licensed under a Creative Commons Attribution 4.0 International License. Read Full License 


\section{Abstract}

Axonal damage in the corpus callosum is prevalent in multiple sclerosis (MS). Although callosal damage is associated with disrupted functional connectivity between hemispheres, it is unclear how this relates to cognitive and physical disability. We investigated this phenomenon using advanced measures of microstructural integrity in the corpus callosum and surface-based Homologous Inter-hemispheric Connectivity (sHIC) in the cortex. We found that sHIC was significantly decreased in primary motor, somatosensory, visual, and temporal cortical areas in a group of 36 participants with MS (29 relapsingremitting, 4 secondary progressive MS, and 3 primary progressive MS) compared with 42 healthy controls (cluster level, $p<0.05$ ). In participants with MS, global sHIC correlated with fractional anisotropy and restricted volume fraction in the posterior segment of the corpus callosum $(r=0.433, p=0.013 ; r=0.325$, $\mathrm{p}=0.021$ ). Lower $\mathrm{sHIC}$, particularly in somatomotor and posterior cortical areas, was associated with cognitive impairment and higher disability scores on the Expanded Disability Status Scale (EDSS). We demonstrated that higher levels of SHIC attenuated the effects of posterior callosal damage on physical disability and cognitive dysfunction, as measured by the EDSS and Brief Visuospatial Memory TestRevised (interaction effect, $\mathrm{p}<0.05$ ). We also observed a positive association between global sHIC and years of education $(r=0.368, p=0.018)$, supporting the phenomenon of "brain reserve" in MS. Our data suggests that preserved SHIC helps prevent cognitive and physical decline in MS.

\section{Introduction}

The corpus callosum (CC) is a critical white-matter tract in the pathophysiology of multiple sclerosis (MS). As the primary inter-hemispheric tract, the CC is particularly vulnerable to damage in MS (Barnard \& Triggs, 1974). This is likely the result of many factors, such as direct focal lesions and Wallerian degeneration from surrounding white and gray matter pathology (Evangelou et al., 2000; Ge et al., 2004; Klawiter et al., 2015). Structural disconnection of the CC is strongly associated with cognitive impairment (Bergendal et al., 2013; Bodini et al., 2013; Granberg et al., 2015; Huang et al., 2019) and physical disability (Llufriu et al., 2012; Ozturk et al., 2010; Tallantyre et al., 2010).

Resting-state functional connectivity may help to elucidate the relationship between CC axonal damage and clinical disability in MS. The CC is essential for facilitating connections between bilateral, homotopic regions (Innocenti, 2009), which generally have high functional connectivity across hemispheres (Biswal et al., 1995). Various methods have been used to investigate how CC damage may influence interhemispheric homotopy in MS. Using EEG, Zito et al. (2014) found that CC atrophy was associated with less efficacious inter-hemispheric coherence in people with relapsing remitting MS (RRMS) when performing a motor task. A task-based fMRI study found that CC atrophy in MS disrupted interhemispheric inhibition in the motor cortex (Manson et al., 2006), and resting-state fMRI work showed that decreased functional connectivity between the bilateral primary sensorimotor cortices in MS was associated with damage in the corresponding transcallosal white matter tract (Lowe et al., 2008). Using the technique of voxel-mirrored homotopic connectivity (VMHC), Zhou et al. (2013) found a positive correlation between $\mathrm{VMHC}$ and fractional anisotropy (FA) of the $\mathrm{CC}$. While these studies demonstrate a 
structure-function relationship, it is unclear how CC damage and homologous connectivity may interact and contribute to disability in MS. Work by Lin et al. (2020) found associations between altered functional inter-hemispheric connectivity and impaired information processing speed, while Zhou et al. (2013) found no correlation between global VMHC and Expanded Disability Status Scale (EDSS) scores.

Improvements in gradient hardware have allowed for the development of new diffusion MRI measures with useful applications in MS. Previous studies comparing diffusion tensor imaging (DTI) measures with MS histopathology have shown that conventional diffusion measures such as radial diffusivity, mean diffusivity (MD), and FA reflect demyelination more than axonal loss (Klawiter et al., 2011; Schmierer et al., 2007). The use of high gradient strengths up to $300 \mathrm{mT} / \mathrm{m}$ in the living human brain has enabled estimation of axonal size and density, providing measures for axonal integrity (Huang et al., 2015; Huang et al., 2020; Veraart et al., 2020). Using this technique, Huang et al. (2019) found that altered apparent axon diameter in the $\mathrm{CC}$ of participants with MS correlated with disability and cognitive dysfunction. These advanced measures may also provide a more sensitive biomarker for detecting the effects of CC damage on inter-hemispheric functional connectivity.

The purpose of this study is to investigate the interactions between CC damage, resting-state functional connectivity, and clinical disability using novel measures of microstructural integrity and interhemispheric connectivity. We evaluated functional connectivity between homologous regions using a technique known as surface-based Homologous Inter-hemispheric Connectivity (sHIC) (Tobyne et al., 2016). Surface-based analysis has proven to be more accurate and spatially specific than volumetric approaches (Anticevic et al., 2008; Jo et al., 2008; Jo et al., 2007; Tucholka et al., 2012), allowing for improved symmetry and mapping of homotopic connections. SHIC has been validated in a large healthy control (HC) dataset and shown to correlate negatively with CC atrophy in MS (Tobyne et al., 2016). Along with the conventional DTI measures of MD and FA, we investigated high gradient diffusion MRI estimates of axon density, apparent axon diameter, and restricted volume fraction in the CC. First, we assessed differences in SHIC between MS and HC groups. We then examined how SHIC relates to CC damage and clinical disability in MS, both independently and combined in a multiple regression analysis. We hypothesized that microstructural alterations in the $\mathrm{CC}$ would disrupt homologous connectivity and contribute to clinical disability in tests dependent upon inter-hemispheric function.

\section{Materials And Methods}

\section{Study Population}

Patients were recruited from the Massachusetts General Hospital Multiple Sclerosis Clinic. This study was conducted on a group of 36 participants with MS (29 RRMS, 4 secondary progressive MS (SPMS), and 3 primary progressive MS (PPMS)). This group (mean age $=43.9 \pm 11.5$, age range $23-60, \mathrm{M}: \mathrm{F} 9: 27$ ) had a mean EDSS of $2.8 \pm 1.8$ (range 1-6.5) and a mean disease duration of $9.7 \pm 6.7$ years (range 1-23). Inclusion criteria for participants with MS were: a diagnosis of clinically definite MS, absence of clinical relapse within 3 months, and being on stable disease-modifying treatment or no treatment for at least 6 
months. Exclusion criteria were: other major medical and/or psychiatric disorders, severe claustrophobia, and presence of MRI contraindications.

The healthy control group used for the resting-state $\mathrm{fMRI}$ analysis consisted of 42 participants (mean age $=37.3 \pm 14.9$, age range 20-63, M:F 14:28). The HC dataset was derived from the MGH-Harvard-USC Lifespan dataset (Fan et al., 2016) and from a separate technical development study using the same scanner. Additionally, a separate group of 59 healthy participants (mean age $=31.5 \pm 9.2$, age range $20-$ 60, M:F 31:18) was taken from the MGH/UCLA Consortium Human Connectome Project (HCP, http://www.humanconnectomeproject.org). This group (referred to as HCP) was used to construct a healthy structural connectome. Informed consent was obtained from all participants.

Neurological disability was measured for all patients with MS using clinical tests included in the Minimal Assessment of Cognitive Function in MS (MACFIMS) battery (Benedict et al., 2006) and Multiple Sclerosis Functional Composite (MSFC) (Cutter et al., 1999). Tests were administered by a trained examiner within one week of the MRI scan. We characterized cognitive performance using the neuropsychological tests BVMT-TR (Brief Visuospatial Memory Test - Total Recall), PASAT (Paced-Auditory-Serial Addition Test - 3 seconds), and SDMT (Symbol Digit Modalities Test). Physical disability was measured using timed 25foot walk and timed 9-hole peg test. A board-certified neurologist conducted a clinical examination and calculated EDSS scores.

\section{MRI Acquisition}

All participants were scanned on the same 3 Tesla MRI scanner (MAGNETOM CONNECTOM, Siemens Healthcare, Erlangen, Germany) with a maximum gradient strength of $300 \mathrm{mT} / \mathrm{m}$ using a customdesigned 64-channel head coil (Keil et al., 2013). High-resolution three-dimensional T1-weighted (T1w) multi-echo magnetization prepared rapid gradient echo (MEMPRAGE) anatomical images (repetition time (TR)/echo time (TE)/inversion time $(\mathrm{TI})=2530 /[1.15,3.03,4.89,6.75] / 1100 \mathrm{~ms}$, field of view $(\mathrm{FOV})=256$, flip angle $(F A)=7^{\circ}, 1 \times 1 \times 1 \mathrm{~mm}^{3}$ voxel resolution, GRAPPA acceleration $(\mathrm{R})=2$ ) were acquired for all participants.

Diffusion data was obtained for the MS and HCP groups. To measure microstructural integrity for the MS participants, a 2D spin-echo EPI sequence (TR/TE $=3600 / 77 \mathrm{~ms}, 2 \times 2 \times 2 \mathrm{~mm}^{3}$ voxel resolution, $\mathrm{R}=2$, slice acceleration factor $=2$ ) was acquired with multi-shell diffusion consisting of two diffusion times $(\Delta$ $=19 \mathrm{~ms}$ and $49 \mathrm{~ms}$ ) and eight gradient strengths per diffusion time, linearly spaced from 30 to $290 \mathrm{mT} / \mathrm{m}$, resulting in a total of $16 \mathrm{~b}$-values for the entire acquisition.

Resting-state fMRI images were acquired for the MS and HC groups (excluding HCP participants) using a 2D single-shot GE EPI sequence sensitive to blood oxygenation level-dependent (BOLD) contrast (TR/TE $=1080 / 30 \mathrm{~ms}, \mathrm{FOV}=220, \mathrm{FA}=60^{\circ}, 2 \times 2 \times 2 \mathrm{~mm}^{3}$ voxel resolution, 68 interleaved slices, 344 measurements, bandwidth $=2840 \mathrm{~Hz} / \mathrm{Px}$, acquisition time $=6.26 \mathrm{~min}$ ). Participants were instructed to keep their eyes open and let their mind wander. 
To construct a healthy structural connectome, a 2D spin-echo EPI sequence (TR/TE $=8800 / 57 \mathrm{~ms}, 1.5 \mathrm{x}$ $1.5 \times 1.5 \mathrm{~mm}^{3}$ voxel resolution, $R=3$ ) with multi-shell diffusion $(b=1,000,64$ directions; $b=5,000,128$ directions; and $\mathrm{b}=10,000,128$ directions) was obtained for all HCP participants.

\section{Data Preprocessing}

Data was preprocessed using the pipeline established for the MGH-USC Human Connectome project (Fan et al., 2016) and tools from FreeSurfer (http://surfer.nmr.mgh.harvard.edu), FMRIB Software Library (FSL, https://fsl.fmrib.ox.ac.uk), MATLAB (version 9.5, Natick, Massachusetts: The MathWorks Inc., 2018b), and custom in-house software. The cortical surface was reconstructed from T1-weighted data using FreeSurfer (version 5.3). An experienced user manually reviewed and edited these reconstructions to correct for artifacts. White matter lesions disrupting the cortical boundary were manually filled in to prevent misclassification of grey matter (Govindarajan et al., 2015).

Functional preprocessing was conducted for each subject volume in native space before being resampled to a symmetric template. Resting state and diffusion-weighted data were corrected for gradient nonlinearities, eddy currents, slice-timing, and motion artifacts, and visually inspected for quality assurance. Functional images were spatially smoothed with a $4 \mathrm{~mm}$ full width half maximum kernel and underwent grand mean intensity scaling and temporal bandpass filtering $(0.001<f<0.08 \mathrm{~Hz})$.

Physiological noise was removed using multiple regression. Resting state data had the following exclusion criteria: 1) functional SNR level < 150; 2) maximal absolute displacement > 1.5mm; 3) average framewise displacement $>0.5 \mathrm{~mm}$; 4 ) more than $5 \%$ of timepoints with a framewise displacement > $0.5 \mathrm{~mm}$; or 5) presence of significant image artifacts. Two resting-state runs were acquired for each subject. If both passed quality assurance, the run with less movement and noise was chosen for analysis.

\section{Surface-based Homotopic Inter-hemispheric Connectivity}

Inter-hemispheric methods (surfer.nmr.mgh.harvard.edu/fswiki/Xhemi) were used to register each hemisphere to the "fsaverage_sym" symmetric surface template developed by FreeSurfer (Greve et al., 2013). sHIC was calculated using in-house MATLAB code and the techniques previously developed and validated by our group (Tobyne et al., 2016). Homologous vertices were defined as pairs of vertices that shared the same spatial location on the symmetric template. We calculated the pairwise Pearson's correlation between the extracted timecourse for each member of each pair of vertices, generating a metric of sHIC per surface vertex. These resulting correlation values were normalized using Fisher's r-to-Z transformation prior to group-level analysis. sHIC correlation maps were represented and analyzed on the left hemisphere.

\section{Corpus Callosum Diffusion Analysis}

The CC was segmented into five evenly spaced sections using the FreeSurfer aparc + aseg atlas, corresponding to the anterior, mid-anterior, central, mid-posterior, and posterior segments of the CC (Fig. 1a). For all MS and HCP participants, each segment was manually edited by a trained research assistant to exclude voxels from the fornix and surrounding cerebrospinal fluid. Axonal integrity was 
measured in the CC using conventional DTI and multi-compartment modeling (Huang et al., 2019; Huang et al., 2020). Apparent axon diameter, restricted volume fraction, and axon density were calculated for each voxel. Apparent axon diameter reflects an estimation based on compartment models of diffusion MRI data and not true axon diameter. Axon density was calculated by weighting the restricted fraction by the cross-sectional area calculated using the mean apparent axon diameter. Diffusion metrics of MD and FA were derived from the DTIFIT tool in FSL using an ordinary least-squares fit to the diffusion MRI data acquired at $b=800 \mathrm{~s} / \mathrm{mm}^{2}$ with a diffusion time of $19 \mathrm{~ms}$ and 32 diffusion-encoding gradient directions. Axon density, apparent axon diameter, restricted volume fraction, FA, and MD were estimated for each CC segment by averaging values over the segmented voxels.

\section{Structural Connectome}

The HCP healthy control dataset was used to construct a generic structural connectome between the segments of the $\mathrm{CC}$ and defined cortical regions. This strategy has been employed in other neurological conditions to provide a more reliable model of connectivity that is not affected by disease pathology (Mandelli et al., 2016; Zhou et al., 2012). The cortical surface was reconstructed from T1-weighted data using FreeSurfer and parcellated into 31 regions per hemisphere using the FreeSurfer generated DesikanKilliany atlas (Fig. 1b). Structural connectivity was measured in each subject using probabilistic streamline tractography. Seed regions for each cortical parcel were defined as the white matter region adjacent to the given parcel. FSL's probtrackx2 (Behrens et al., 2007) was used to perform tractography with 5000 streamlines generated per voxel in the seed ROI, 2000 steps per streamline, and step length of $0.5 \mathrm{~mm}$. The number of samples connecting each pair of regions were entered into a structural connectivity matrix. Each subject's structural connectivity matrix was binarized at a density threshold of $30 \%$ to exclude spurious links (Rubinov \& Sporns, 2010). This was based off of matrix density thresholds used in other studies, which generally range from 10-35\% (Kamagata et al., 2019; Zhang et al., 2011). Subject matrices were combined into a single aggregate matrix containing connections present in at least $50 \%$ of subjects. Based on this final connectivity matrix, each CC segment was associated with a group of structurally connected cortical regions to be investigated using sHIC analysis.

\section{Statistical Analysis}

Statistical calculations were conducted using MATLAB and SPSS (version 27.0. Armonk, NY: IBM Corp). Global sHIC was compared between MS and HC groups using a Student's t-test. sHIC was compared between MS and HC groups and correlated with EDSS scores using a vertex-wise general linear model from the FreeSurfer group analysis pipeline, controlling for age and gender. Correction for multiple comparisons was performed at the cluster level using Monte Carlo simulation (Hagler et al., 2006). sHIC was also extracted from defined cortical regions and correlated with $\mathrm{CC}$ diffusion measures and clinical test scores using a pairwise Pearson correlation and a multiple linear regression model to adjust $p$-values for age and gender. Additionally, multiple linear regression was used to assess the interaction between sHIC and CC damage in predicting clinical disability, while controlling for age, gender, and years of education. 


\section{Results}

\section{Group Differences in sHIC}

Average sHIC correlation maps for MS and HC groups show that inter-hemispheric connectivity was highest in primary motor, somatosensory, and visual cortices, consistent with previous studies (Fig. 2). Participants with MS exhibited decreased SHIC in comparison to healthy controls. Global SHIC, defined as a measure of the mean sHIC throughout the whole cortex, was significantly reduced in the MS group $(0.242 \pm 0.065)$ compared to the HC group $(0.284 \pm 0.062)(p=0.004)$. This was further explored using a vertex-wise group analysis, which showed that SHIC was decreased in several cortical areas in the MS group based on vertex and cluster-wise significance thresholds of $p<0.01$ and $p<0.05$, respectively (Fig. 2). These alterations were most pronounced in primary motor, somatosensory, visual, cuneus, and temporal cortical areas (Table 1). There were no areas where sHIC was significantly higher in the MS group.

\section{sHIC and Diffusion Measures in the Corpus Callosum}

The strongest associations between SHIC and CC diffusion measures were found in the posterior segment of the CC (Table 2). Average sHIC was extracted from each cortical region structurally connected to the posterior $\mathrm{CC}$ and correlated with each diffusion metric in the posterior $\mathrm{CC}$, controlling for age and gender. sHIC extracted from individual cortical regions, as parcellated by the Desikan-Killiany atlas, correlated most strongly with FA and restricted volume fraction in the posterior $\mathrm{CC}$. Correlations between sHIC and axon diameter, axon density, and MD in the posterior CC were generally weak or did not survive correction for age and gender. Average sHIC extracted over all cortical regions connected to the posterior CC (which we will refer to as posterior SHIC) correlated with FA in the posterior $C C(r=0.401, p=0.024)$. Global sHIC also significantly correlated with FA $(r=0.433, p=0.013)$ and restricted volume fraction $(r=$ $0.325, p=0.021$ ) in the posterior CC (Fig. 3).

Measures of cortical sHIC did not correlate well with diffusion measures in the anterior, mid-anterior, central, and mid-posterior segments of the CC. The only significant correlation found was between mean sHIC in caudal middle frontal cortex and FA/MD in the mid-posterior $\mathrm{CC}$, controlling for age and gender $(r$ $=0.372, p=0.029 ; r=-0.396, p=0.016)$. Otherwise, there were no significant associations between sHIC and diffusion measures in these four CC segments. Also, there were no correlations between global sHIC and any diffusion measures extracted from the whole CC.

\section{sHIC and Clinical Outcomes}

Cortical regions with significant correlations between mean sHIC and clinical test scores, controlling for age and gender, are reported in Table 3. For several cortical regions, reduced sHIC correlated with poor performance on tests of visual learning and memory (BVMT-TR) and information processing speed (SDMT and PASAT), slower 25-foot walk and 9-hole peg test times, and higher overall disability as measured by EDSS. There were no correlations between global SHIC and any of the clinical measures. We also investigated EDSS using a vertex-wise general linear model in the MS group, controlling for age and 
gender, applying vertex and cluster-wise significance thresholds of $p<0.05$ (Fig. 4). Significant clusters associating decreased sHIC with elevated EDSS were found in several regions, notably motor and somatosensory cortex. We also observed that years of education correlated with global sHIC $(r=0.368, p$ $=0.018)$ and posterior SHIC $(r=0.354, p=0.020)$ in MS, controlling for age and gender .

\section{sHIC Interaction Effects}

We sought to determine if SHIC moderates the effect of CC structural damage on clinical disability by applying multiple linear regression analysis. Based on the previous results from this study, we investigated restricted volume fraction and FA in the posterior CC, and its interaction with posterior sHIC. Each regression model predicted a clinical outcome and contained covariates for age, gender, years of education, posterior $\mathrm{CC}$ microstructural damage, posterior SHIC, and an interaction term between posterior $\mathrm{CC}$ damage and SHIC. A statistically significant interaction term would suggest that the effect of posterior CC damage on clinical function depends on a subject's SHIC. All $\beta$-coefficients reported are standardized. For the regression model predicting EDSS, we observed a significant effect of age $(\beta=0.522, p=0.002)$, sHIC $(\beta=-2.763, p=0.018)$, restricted volume fraction $(\beta=-1.768, p=0.007)$, and the restricted volume fraction/sHIC interaction term ( $\beta=3.381, p=0.021)$ (Fig. $5 a)$. In an additional model for EDSS, significant effects were found for age $(\beta=0.359, p=0.023)$, sHIC $(\beta=-4.224, p=0.015)$, FA $(\beta=-1.498, p=0.006)$, and the FA/sHIC interaction term $(\beta=4.799, p=0.017)$ (Fig. $5 c)$. For the model predicting BVMT performance, a statistically significant effect was found for age $(\beta=-0.536, p=0.001)$, sHIC $(\beta=3.246, p$ $=0.005)$, restricted volume fraction $(\beta=1.707, p=0.007)$, and the restricted volume fraction $/ \mathrm{sHIC}$ interaction term $(\beta=-3.774, p=0.009)$ (Fig. $5 b)$. In an additional model for BVMT, significant effects were found for age $(\beta=-0.441, p=0.005)$, sHIC $(\beta=4.802, p=0.005)$, FA $(\beta=1.335, p=0.011)$, and the $F A / s H I C$ interaction term $(\beta=-5.245, p=0.008)$ (Fig. $5 d$ ). Interaction terms were not significant for PASAT, SDMT, or 9-hole-peg test. Overall these findings suggest that in participants with MS, the relationship between posterior CC damage and clinical disability, as measured by EDSS and BVMT, is partly dependent on the level of posterior sHIC.

\section{Discussion}

In this study, we investigated how functional inter-hemispheric connectivity relates to structural CC damage and clinical disability in MS. We found that decreased SHIC, generally in posterior cortical areas, correlates with structural abnormalities in the posterior $\mathrm{CC}$, cognitive dysfunction, and physical disability. We also showed that MS participants with higher SHIC may have some preserved clinical function as measured by BVMT and EDSS, despite CC damage. These results support our hypothesis that callosal damage is associated with alterations in SHIC that contribute to disability.

Our results demonstrated that SHIC is significantly different in MS compared to HC participants. The patterns of sHIC observed in the MS and HC group average correlation maps indicate that interhemispheric connectivity was strongest in somatomotor and visual cortices, replicating previous work done by our lab and others (Stark et al., 2008; Tobyne et al., 2016; Zhou et al., 2013). We found that these regions were especially vulnerable to alterations in sHIC in participants with MS. sHIC was globally lower 
in the MS group, with the largest local reductions occurring in primary motor, somatosensory, visual, cuneus, and temporal cortical areas. While previous studies have also found reduced homologous connectivity in these regions (Lowe et al., 2008; Zhou et al., 2013), they did not demonstrate group differences in precentral, paracentral, superior frontal, and superior parietal cortical areas. Our more robust detection of group differences may be partly due to the superior accuracy of surface-based registration in comparison to volume-based registration methods (Fischl et al., 2008). It is notable that there were no cortical areas with significantly increased SHIC in the MS group. This is in contrast to other studies that have observed complex patterns of both increased and decreased functional connectivity when evaluating inter-hemispheric or network differences in MS and HC groups (Pasqua et al., 2020; Tona et al., 2014; Zhou et al., 2013). Our findings are consistent with the interpretation that callosal damage reduces transmission and synchrony between homologous regions.

In an unbiased general linear model, we found a significant relationship between increased EDSS scores and reduced sHIC in various clusters located in somatomotor cortex. These clusters also roughly overlapped with those observed in primary somatosensory cortex from the MS versus HC group difference analysis. Because inter-hemispheric connectivity tends to be highest in primary sensory regions, sHIC may be a sensitive tool to better understand sensory dysfunction in MS. While we did not observe any significant associations between global homologous connectivity and clinical disability, lower posterior SHIC did correlate with cognitive impairment, as measured by BVMT, PASAT, and SDMT. We also found that impaired performances on BVMT, PASAT, SDMT, 25-foot walk, and 9-hole peg test were associated with lower SHIC in many cortical regions, particularly in the parietal and occipital lobes. There were no cortical regions for which higher sHIC correlated with disability. Overall, our results support the hypothesis that reduced inter-hemispheric connectivity is predictive of poor clinical outcomes.

We found that the structure-function relationship between $\mathrm{CC}$ axonal integrity and sHIC was most evident in the posterior CC. This may be due to the anterior to posterior gradient previously observed by Tobyne et al. (2016) in a large healthy control dataset, in which posterior nodes of multiple functional networks generally had higher sHIC than the anterior regions. This phenomenon may help explain the lack of significant correlations between diffusion measures in the other four segments of the $\mathrm{CC}$ and the SHIC extracted from their more anterior cortical connections. While the CC is a major white matter bundle connecting the two hemispheres, our tissue microstructure measures did not account for potential changes in hemispheric white matter lateral to the $\mathrm{CC}$. We found that FA and restricted volume fraction in the posterior CC correlated with global SHIC, posterior SHIC, and local SHIC extracted from individual regions in parietal, temporal, and occipital cortex. These results support findings from Zhou et al. (2013), who reported no significant correlations between VHMC and FA for CC segments 1-4, but did find a positive correlation between occipital VHMC and mean FA in the posterior CC (segment 5) in participants with MS. Our results build upon our previous findings that CC atrophy correlates with global sHIC (Tobyne et al., 2016) by adding more specific outcomes for axonal pathology derived from multi-compartmental modeling of the diffusion MRI signal. 
Although sHIC correlated with restricted volume fraction, it was not strongly associated with axon density and apparent axon diameter in the CC. It is notable that while the correlations between SHIC and these axonal imaging measures failed to reach statistical significance after controlling for age and gender, they trended in a meaningful direction. High apparent axon diameter (a marker of damage due to the vulnerability of small diameter axons) and low axon density in the CC have been observed in previous high-gradient diffusion MRI studies of MS (Huang et al., 2019; Huang et al., 2016) and trended with reduced SHIC in many cortical regions. While not statistically significant, our results suggest that it may be useful to evaluate these metrics longitudinally and in a larger group of people with MS. Of the diffusion metrics we investigated in the CC, FA and restricted volume fraction were most highly correlated with inter-hemispheric connectivity. This is consistent with previous studies that looked at FA in the CC. Zhou et al. (2013) found that global VMHC correlated with average FA of the entire CC and Lowe et al. (2008) found that decreased inter-hemispheric functional connectivity was associated with decreased FA in transcallosal white matter, but both of these results were only significant in combined MS and HC groups. Using SHIC, we demonstrated this relationship between homologous connectivity and CC damage in the MS group alone.

It is well established that CC damage contributes to clinical disability in MS, however it has been unclear what role inter-hemispheric functional connectivity plays in this relationship. We found that higher levels of sHIC may attenuate the effects of structural CC damage on disability. This was demonstrated through a post-hoc investigation in the posterior $\mathrm{CC}$, looking at restricted volume fraction, FA, and posterior sHIC due to their prior significance. We found that in MS participants with higher average SHIC, alterations in CC microstructure were less associated with clinical impairment, as measured by EDSS and BVMT scores. Increased functional connectivity in MS is a complex phenomenon that has been associated with both clinical improvement (Fuchs et al., 2019; Penner \& Aktas, 2017; Sumowski et al., 2013) and decline (Hawellek et al., 2011; Rocca \& Filippi, 2017). In this context, high measures of sHIC might indicate that inter-hemispheric functional networks are more adaptable or resilient to microstructural damage in the CC. This compensatory effect may help to preserve cognitive and physical ability. This is consistent with previous studies which have found that MS participants with more normal patterns of default mode network activity were more protected against atrophy-related memory impairment (Sumowski et al., 2013), and MS participants with preserved functional connectivity in cognitive networks maintained cognitive capacity despite grey matter atrophy and white matter tract disruption (Fuchs et al., 2019). We also found that global sHIC for MS participants positively correlated with years of education, which has generally been thought to contribute to "brain reserve" in neurodegenerative disorders (Nithianantharajah \& Hannan, 2009). This suggests that environmental factors like education may help preserve interhemispheric functional connectivity and prevent progression of disability.

\section{Limitations}

This study was conducted on a group of participants with relapsing-remitting and progressive MS. The sample size of the progressive MS subgroup was too small to perform a meaningful sub-analysis. While combining these groups provided more statistical power, it limits the conclusions for specific MS 
phenotypes. Additionally, the cross-sectional nature of this study helps reveal associations between sHIC, CC damage, and disability, but a longitudinal study would be necessary to determine if sHIC plays a causal role in preserving clinical function as structural damage accumulates in the brain.

Although sHIC improves spatial accuracy, the technique is limited to the cortical surface. Therefore, the relevant effects of inter-hemispheric connectivity in subcortical and cerebellar regions are not considered. Future work to expand the symmetric template to include subcortical structures would alleviate this issue. Similarly, our analysis of structural damage was limited to diffusion measures in the CC. This fails to account for lesions, atrophy, and microstructural alterations outside of the CC, which may influence interhemispheric connectivity

\section{Declarations}

\section{Funding:}

This work was supported by the National Institutes of Health (K23NS096056 to S.Y.H., K23NS078044 to E.C.K., R00EB015445 to A.N.), the National Multiple Sclerosis Society (E.C.K.), the Radiological Society of North America and Conrad N. Hilton Foundation (S.Y.H.).

\section{Conflicts of interest/Competing interests:}

ECK has received consulting fees from Alexion, Banner Life Sciences, Biogen, EMD Serono, Genentech, and MedDay and research funding from Abbvie, Biogen, EMD Serono, Genentech/Roche, and Genzyme. All other authors have no relevant financial or non-financial interests to disclose.

\section{Availability of data and material:}

The datasets generated and analyzed during the current study are available from the corresponding author on reasonable request. Healthy control data from the Human Connectome Project and the MGHHarvard-USC Lifespan Dataset are publicly available through the Laboratory of Neuro Imaging Image Data Archive (https://ida.loni.usc.edu) and the WU-Minn Connectome Database (https://db.humanconnectome.org).

\section{Code availability:}

Code is available upon request.

\section{Authors' contributions:}


Conceptualization: Eric C. Klawiter, Andrew W. Russo; Data acquisition, analysis, and manuscript preparation: Andrew W. Russo, Kirsten E. Stockel, Sean M. Tobyne, Chanon Ngamsombat, Kristina Brewer, Aapo Nummenmaa, Susie Y. Huang, Eric C. Klawiter

\section{Ethics approval:}

The study was approved by the Mass General Brigham institutional review board and is in accordance with the 1964 Helsinki Declaration.

\section{Consent to participate:}

Informed consent was obtained from all individual participants included in the study.

\section{Consent for publication:}

All subjects provided written informed consent for publication of data.

\section{Acknowledgements:}

Data collection and sharing for this project was provided in part by the MGH-USC Human Connectome Project (HCP; Principal Investigators: Bruce Rosen, M.D., Ph.D., Arthur W. Toga, Ph.D., Van J. Weeden, MD). HCP funding was provided by the National Institute of Dental and Craniofacial Research (NIDCR), the National Institute of Mental Health (NIMH), and the National Institute of Neurological Disorders and Stroke (NINDS).

\section{References}

1. Anticevic A, Dierker DL, Gillespie SK, Repovs G, Csernansky JG, Van Essen DC, Barch DM (2008) Comparing surface-based and volume-based analyses of functional neuroimaging data in patients with schizophrenia. Neuroimage 41(3):835-848. https://doi.org/10.1016/j.neuroimage.2008.02.052

2. Barnard RO, Triggs M (1974) Corpus callosum in multiple sclerosis. J Neurol Neurosurg Psychiatry 37(11):1259-1264. https://doi.org/10.1136/jnnp.37.11.1259

3. Behrens TE, Berg HJ, Jbabdi S, Rushworth MF, Woolrich MW (2007) Probabilistic diffusion tractography with multiple fibre orientations: What can we gain? Neuroimage 34(1):144-155. https://doi.org/10.1016/j.neuroimage.2006.09.018

4. Benedict RH, Cookfair D, Gavett R, Gunther M, Munschauer F, Garg N, Weinstock-Guttman B (2006) Validity of the minimal assessment of cognitive function in multiple sclerosis (MACFIMS). J Int Neuropsychol Soc 12(4):549-558. https://doi.org/10.1017/s1355617706060723 
5. Bergendal G, Martola J, Stawiarz L, Kristoffersen-Wiberg M, Fredrikson S, Almkvist O (2013) Callosal atrophy in multiple sclerosis is related to cognitive speed. Acta Neurol Scand 127(4):281-289. https://doi.org/10.1111/ane.12006

6. Biswal B, Yetkin FZ, Haughton VM, Hyde JS (1995) Functional connectivity in the motor cortex of resting human brain using echo-planar MRI. Magn Reson Med 34(4):537-541. https://doi.org/10.1002/mrm.1910340409

7. Bodini B, Cercignani M, Khaleeli Z, Miller DH, Ron M, Penny S,.. . Ciccarelli O (2013) Corpus callosum damage predicts disability progression and cognitive dysfunction in primary-progressive MS after five years. Hum Brain Mapp 34(5):1163-1172. https://doi.org/10.1002/hbm.21499

8. Cutter GR, Baier ML, Rudick RA, Cookfair DL, Fischer JS, Petkau J,.. . Willoughby E (1999) Development of a multiple sclerosis functional composite as a clinical trial outcome measure. Brain, 122 (Pt 5), 871-882

9. Evangelou N, Konz D, Esiri MM, Smith S, Palace J, Matthews PM (2000) Regional axonal loss in the corpus callosum correlates with cerebral white matter lesion volume and distribution in multiple sclerosis. Brain, 123 (Pt 9), 1845-1849

10. Fan Q, Witzel T, Nummenmaa A, Van Dijk KRA, Van Horn JD, Drews MK,... Rosen BR (2016) MGHUSC Human Connectome Project datasets with ultra-high b-value diffusion MRI. Neuroimage 124(Pt B), 1108-1114. https://doi.org/10.1016/j.neuroimage.2015.08.075

11. Fischl B, Rajendran N, Busa E, Augustinack J, Hinds O, Yeo BT,.. . Zilles K (2008) Cortical folding patterns and predicting cytoarchitecture. Cereb Cortex 18(8):1973-1980. https://doi.org/10.1093/cercor/bhm225

12. Fuchs TA, Benedict RHB, Bartnik A, Choudhery S, Li X, Mallory M,... Dwyer MG (2019) Preserved network functional connectivity underlies cognitive reserve in multiple sclerosis. Hum Brain Mapp 40(18):5231-5241. https://doi.org/10.1002/hbm.24768

13. Ge Y, Law M, Johnson G, Herbert J, Babb JS, Mannon LJ, Grossman RI (2004) Preferential occult injury of corpus callosum in multiple sclerosis measured by diffusion tensor imaging. J Magn Reson Imaging 20(1):1-7. https://doi.org/10.1002/jmri.20083

14. Govindarajan KA, Datta S, Hasan KM, Choi S, Rahbar MH, Cofield SS,.. . Group Cl (2015) Effect of inpainting on cortical thickness measurements in multiple sclerosis: A large cohort study. Hum Brain Mapp 36(10):3749-3760. https://doi.org/10.1002/hbm.22875

15. Granberg T, Martola J, Bergendal G, Shams S, Damangir S, Aspelin P,.. . Kristoffersen-Wiberg M (2015) Corpus callosum atrophy is strongly associated with cognitive impairment in multiple sclerosis: Results of a 17-year longitudinal study. Mult Scler 21(9):1151-1158. https://doi.org/10.1177/1352458514560928

16. Greve DN, Van der Haegen L, Cai Q, Stufflebeam S, Sabuncu MR, Fischl B, Brysbaert M (2013) A surface-based analysis of language lateralization and cortical asymmetry. J Cogn Neurosci 25(9):1477-1492. https://doi.org/10.1162/jocn_a_00405 
17. Hagler DJ, Saygin AP, Sereno MI (2006) Smoothing and cluster thresholding for cortical surfacebased group analysis of fMRI data. Neuroimage 33(4):1093-1103.

https://doi.org/10.1016/j.neuroimage.2006.07.036

18. Hawellek DJ, Hipp JF, Lewis CM, Corbetta M, Engel AK (2011) Increased functional connectivity indicates the severity of cognitive impairment in multiple sclerosis. Proc Natl Acad Sci U S A 108(47):19066-19071. https://doi.org/10.1073/pnas.1110024108

19. Huang SY, Fan Q, Machado N, Eloyan A, Bireley JD, Russo AW,.. . Klawiter EC (2019) Corpus callosum axon diameter relates to cognitive impairment in multiple sclerosis. Ann Clin Transl Neurol 6(5):882892. https://doi.org/10.1002/acn3.760

20. Huang SY, Nummenmaa A, Witzel T, Duval T, Cohen-Adad J, Wald LL, McNab JA (2015) The impact of gradient strength on in vivo diffusion MRI estimates of axon diameter [Research Support. N.I.H., Extramural

21. Research Support Non-US, Gov't]. Neuroimage, 106, 464-472. https://doi.org/10.1016/j.neuroimage.2014.12.008

22. Huang SY, Tian Q, Fan Q, Witzel T, Wichtmann B, McNab JA,.. . Nummenmaa A (2020) High-gradient diffusion MRI reveals distinct estimates of axon diameter index within different white matter tracts in the in vivo human brain. Brain Struct Funct 225(4):1277-1291. https://doi.org/10.1007/s00429-01901961-2

23. Huang SY, Tobyne SM, Nummenmaa A, Witzel T, Wald LL, McNab JA, Klawiter EC (2016) Characterization of Axonal Disease in Patients with Multiple Sclerosis Using High-Gradient-Diffusion MR Imaging. Radiology, 151582. https://doi.org/10.1148/radiol.2016151582

24. Innocenti GM (2009) Dynamic interactions between the cerebral hemispheres. Exp Brain Res 192(3):417-423. https://doi.org/10.1007/s00221-008-1484-8

25. Jo HJ, Lee JM, Kim JH, Choi CH, Gu BM, Kang DH,.. . Kim SI (2008) Artificial shifting of fMRI activation localized by volume- and surface-based analyses. Neuroimage 40(3):1077-1089. https://doi.org/10.1016/j.neuroimage.2007.12.036

26. Jo HJ, Lee JM, Kim JH, Shin YW, Kim IY, Kwon JS, Kim SI (2007) Spatial accuracy of fMRI activation influenced by volume- and surface-based spatial smoothing techniques. Neuroimage 34(2):550564. https://doi.org/10.1016/j.neuroimage.2006.09.047

27. Kamagata K, Zalesky A, Yokoyama K, Andica C, Hagiwara A, Shimoji K,.. . Aoki S (2019) MR g-ratioweighted connectome analysis in patients with multiple sclerosis. Sci Rep 9(1):13522. https://doi.org/10.1038/s41598-019-50025-2

28. Keil B, Blau JN, Biber S, Hoecht P, Tountcheva V, Setsompop K,.. . Wald LL (2013) A 64-channel 3T array coil for accelerated brain MRI. Magn Reson Med 70(1):248-258. https://doi.org/10.1002/mrm.24427

29. Klawiter EC, Ceccarelli A, Arora A, Jackson J, Bakshi S, Kim G,.. . Neema M (2015) Corpus callosum atrophy correlates with gray matter atrophy in patients with multiple sclerosis. J Neuroimaging 25(1):62-67. https://doi.org/10.1111/jon.12124 
30. Klawiter EC, Schmidt RE, Trinkaus K, Liang HF, Budde MD, Naismith RT,.. . Benzinger TL (2011) Radial diffusivity predicts demyelination in ex vivo multiple sclerosis spinal cords. Neuroimage 55(4):14541460. https://doi.org/10.1016/j.neuroimage.2011.01.007

31. Lin SJ, Kolind S, Liu A, McMullen K, Vavasour I, Wang ZJ,.. . McKeown MJ (2020) Both Stationary and Dynamic Functional Interhemispheric Connectivity Are Strongly Associated With Performance on Cognitive Tests in Multiple Sclerosis. Front Neurol 11:407. https://doi.org/10.3389/fneur.2020.00407

32. Llufriu S, Blanco Y, Martinez-Heras E, Casanova-Molla J, Gabilondo I, Sepulveda M,.. . Saiz A (2012) Influence of corpus callosum damage on cognition and physical disability in multiple sclerosis: a multimodal study. PLoS One 7(5):e37167. https://doi.org/10.1371/journal.pone.0037167

33. Lowe MJ, Beall EB, Sakaie KE, Koenig KA, Stone L, Marrie RA, Phillips MD (2008) Resting state sensorimotor functional connectivity in multiple sclerosis inversely correlates with transcallosal motor pathway transverse diffusivity. Hum Brain Mapp 29(7):818-827. https://doi.org/10.1002/hbm.20576

34. Mandelli ML, Vilaplana E, Brown JA, Hubbard HI, Binney RJ, Attygalle S,.. . Gorno-Tempini ML (2016) Healthy brain connectivity predicts atrophy progression in non-fluent variant of primary progressive aphasia. Brain 139(Pt 10):2778-2791. https://doi.org/10.1093/brain/aww195

35. Manson SC, Palace J, Frank JA, Matthews PM (2006) Loss of interhemispheric inhibition in patients with multiple sclerosis is related to corpus callosum atrophy. Exp Brain Res 174(4):728-733. https://doi.org/10.1007/s00221-006-0517-4

36. Nithianantharajah J, Hannan AJ (2009) The neurobiology of brain and cognitive reserve: mental and physical activity as modulators of brain disorders. Prog Neurobiol 89(4):369-382. https://doi.org/10.1016/j.pneurobio.2009.10.001

37. Ozturk A, Smith SA, Gordon-Lipkin EM, Harrison DM, Shiee N, Pham DL,.. . Reich DS (2010) MRI of the corpus callosum in multiple sclerosis: association with disability. Mult Scler 16(2):166-177. https://doi.org/10.1177/1352458509353649

38. Pasqua G, Tommasin S, Bharti K, Ruggieri S, Petsas N, Piervincenzi C,.. . Pantano P (2020) Restingstate functional connectivity of anterior and posterior cerebellar lobes is altered in multiple sclerosis. Mult Scler, 1352458520922770. https://doi.org/10.1177/1352458520922770

39. Penner IK, Aktas O (2017) Functional reorganization is a maladaptive response to injury - NO. Mult Scler 23(2):193-194. https://doi.org/10.1177/1352458516679895

40. Rocca MA, Filippi M (2017) Functional reorganization is a maladaptive response to injury - YES. Mult Scler 23(2):191-193. https://doi.org/10.1177/1352458516667242

41. Rubinov M, Sporns O (2010) Complex network measures of brain connectivity: uses and interpretations. Neuroimage 52(3):1059-1069. https://doi.org/10.1016/j.neuroimage.2009.10.003

42. Schmierer K, Wheeler-Kingshott CA, Boulby PA, Scaravilli F, Altmann DR, Barker GJ,.. . Miller DH (2007) Diffusion tensor imaging of post mortem multiple sclerosis brain. Neuroimage 35(2):467477. https://doi.org/10.1016/j.neuroimage.2006.12.010 
43. Stark DE, Margulies DS, Shehzad ZE, Reiss P, Kelly AM, Uddin LQ,.. . Milham MP (2008) Regional variation in interhemispheric coordination of intrinsic hemodynamic fluctuations. J Neurosci 28(51):13754-13764. https://doi.org/10.1523/JNEUROSCI.4544-08.2008

44. Sumowski JF, Wylie GR, Leavitt VM, Chiaravalloti ND, DeLuca J (2013) Default network activity is a sensitive and specific biomarker of memory in multiple sclerosis. Mult Scler 19(2):199-208. https://doi.org/10.1177/1352458512448267

45. Tallantyre EC, Bo L, Al-Rawashdeh O, Owens T, Polman CH, Lowe JS, Evangelou N (2010) Clinicopathological evidence that axonal loss underlies disability in progressive multiple sclerosis. Mult Scler 16(4):406-411. https://doi.org/10.1177/1352458510364992

46. Tobyne SM, Boratyn D, Johnson JA, Greve DN, Mainero C, Klawiter EC (2016) A surface-based technique for mapping homotopic interhemispheric connectivity: Development, characterization, and clinical application. Hum Brain Mapp 37(8):2849-2868. https://doi.org/10.1002/hbm.23214

47. Tona F, Petsas N, Sbardella E, Prosperini L, Carmellini M, Pozzilli C, Pantano P (2014) Multiple sclerosis: altered thalamic resting-state functional connectivity and its effect on cognitive function. Radiology 271(3):814-821. https://doi.org/10.1148/radiol.14131688

48. Tucholka A, Fritsch V, Poline JB, Thirion B (2012) An empirical comparison of surface-based and volume-based group studies in neuroimaging. Neuroimage 63(3):1443-1453. https://doi.org/10.1016/j.neuroimage.2012.06.019

49. Veraart J, Nunes D, Rudrapatna U, Fieremans E, Jones DK, Novikov DS, Shemesh N (2020) Nonivasive quantification of axon radii using diffusion MRI. Elife, 9. https://doi.org/10.7554/eLife.49855

50. Zhang J, Wang J, Wu Q, Kuang W, Huang X, He Y, Gong Q (2011) Disrupted brain connectivity networks in drug-naive, first-episode major depressive disorder. Biol Psychiatry 70(4):334-342. https://doi.org/10.1016/j.biopsych.2011.05.018

51. Zhou J, Gennatas ED, Kramer JH, Miller BL, Seeley WW (2012) Predicting regional neurodegeneration from the healthy brain functional connectome. Neuron 73(6):1216-1227. https://doi.org/10.1016/j.neuron.2012.03.004

52. Zhou Y, Milham M, Zuo XN, Kelly C, Jaggi H, Herbert J,.. . Ge Y (2013) Functional homotopic changes in multiple sclerosis with resting-state functional MR imaging. AJNR Am J Neuroradiol 34(6):11801187. https://doi.org/10.3174/ajnr.A3386

53. Zito G, Luders E, Tomasevic L, Lupoi D, Toga AW, Thompson PM,... Tecchio F (2014) Interhemispheric functional connectivity changes with corpus callosum morphology in multiple sclerosis. Neuroscience 266:47-55. https://doi.org/10.1016/j.neuroscience.2014.01.039

\section{Tables}


Table 1

Cortical regions with areas of decreased SHIC in participants with MS

\begin{tabular}{|c|c|c|}
\hline Cortical Region & Cluster sizes $\left(\mathrm{mm}^{2}\right)$ & $\begin{array}{l}\text { Cluster-wise } \\
\text { p-values }\end{array}$ \\
\hline Cuneus & $288^{\star *}$ & 0.0001 \\
\hline Fusiform & 64 & 0.011 \\
\hline Inferior temporal & 58 & 0.020 \\
\hline Lateral occipital & $123^{*}$ & 0.001 \\
\hline Lingual & $81^{*}$ & 0.002 \\
\hline Middle temporal & $127^{\star \star}, 137 * \star, 66^{\star}$ & $0.0001,0.0001,0.009$ \\
\hline Paracentral & $349 * \star$ & 0.0001 \\
\hline Parsopercularis & 55 & 0.029 \\
\hline Pericalcerine & $124^{\star \star}$ & 0.0001 \\
\hline Postcentral & $290 * *, 71 *, 54$ & $0.0001,0.004,0.036$ \\
\hline Precentral & $233^{\star \star}, 188^{\star \star}$ & $0.0002,0.0001$ \\
\hline Precuneus & $94 * \star, 124 * \star$ & $0.0007,0.0001$ \\
\hline Superior frontal & 51,55 & $0.048,0.031$ \\
\hline Superior parietal & $170 * \star$ & 0.0001 \\
\hline Superior temporal & $841^{* *}, 64$ & $0.0001,0.011$ \\
\hline Supramarginal & $96 * \star$ & 0.0006 \\
\hline${ }^{*} \mathrm{p}<0.01,{ }^{* *} \mathrm{p}<0.00$ & & \\
\hline
\end{tabular}


Table 2

Correlations between SHIC and diffusion measures in the posterior CC

\begin{tabular}{|c|c|c|c|c|c|}
\hline $\begin{array}{l}\text { Mean sHIC extracted from } \\
\text { cortical regions }\end{array}$ & $\begin{array}{l}\text { Restricted volume } \\
\text { fraction }\end{array}$ & FA & MD & $\begin{array}{l}\text { Axon } \\
\text { diameter }\end{array}$ & $\begin{array}{l}\text { Axon } \\
\text { density }\end{array}$ \\
\hline Caudal anterior cingulate & $0.152(0.609)$ & $\begin{array}{l}0.357 \\
(0.023) *\end{array}$ & $\begin{array}{l}-0.131 \\
(0.174)\end{array}$ & $\begin{array}{l}-0.082 \\
(0.624)\end{array}$ & $\begin{array}{l}0.088 \\
(0.799)\end{array}$ \\
\hline Inferior parietal & $0.217(0.085)$ & $\begin{array}{l}0.364 \\
(0.042) *\end{array}$ & $\begin{array}{l}-0.373 \\
(0.068)\end{array}$ & $\begin{array}{l}-0.208 \\
(0.228)\end{array}$ & $\begin{array}{l}0.231 \\
(0.098)\end{array}$ \\
\hline Inferior temporal & $0.357(0.017) *$ & $\begin{array}{l}0.405 \\
(0.019) *\end{array}$ & $\begin{array}{l}-0.181 \\
(0.349)\end{array}$ & $\begin{array}{l}-0.029 \\
(0.844)\end{array}$ & $\begin{array}{l}0.201 \\
(0.177)\end{array}$ \\
\hline Lateral occipital & $0.293(0.048) *$ & $\begin{array}{l}0.369 \\
(0.043) *\end{array}$ & $\begin{array}{l}-0.092 \\
(0.975)\end{array}$ & $\begin{array}{l}-0.237 \\
(0.215)\end{array}$ & $\begin{array}{l}0.275 \\
(0.101)\end{array}$ \\
\hline Parahippocampal & $0.136(0.533)$ & $\begin{array}{l}0.209 \\
(0.190)\end{array}$ & $\begin{array}{l}-0.278 \\
(0.029) *\end{array}$ & $\begin{array}{l}-0.123 \\
(0.427)\end{array}$ & $\begin{array}{l}0.194 \\
(0.271)\end{array}$ \\
\hline Posterior cingulate & $0.222(0.261)$ & $\begin{array}{l}0.368 \\
(0.032) *\end{array}$ & $\begin{array}{l}-0.177 \\
(0.259)\end{array}$ & $\begin{array}{l}-0.134 \\
(0.491)\end{array}$ & $\begin{array}{l}0.138 \\
(0.544)\end{array}$ \\
\hline Precentral & $0.261(0.049) *$ & $\begin{array}{l}0.382 \\
(0.031) *\end{array}$ & $\begin{array}{l}-0.221 \\
(0.360)\end{array}$ & $\begin{array}{l}-0.081 \\
(0.646)\end{array}$ & $\begin{array}{l}0.179 \\
(0.190)\end{array}$ \\
\hline Superior parietal & $0.405(0.016) *$ & $\begin{array}{l}0.516 \\
(0.002) *\end{array}$ & $\begin{array}{l}-0.339 \\
(0.066)\end{array}$ & $\begin{array}{l}-0.211 \\
(0.292)\end{array}$ & $\begin{array}{l}0.348 \\
(0.060)\end{array}$ \\
\hline Whole cortex (global) & $0.325(0.021)^{*}$ & $\begin{array}{l}0.433 \\
(0.013) *\end{array}$ & $\begin{array}{l}-0.247 \\
(0.285)\end{array}$ & $\begin{array}{l}-0.093 \\
(0.623)\end{array}$ & $\begin{array}{l}0.223 \\
(0.134)\end{array}$ \\
\hline $\begin{array}{l}\text { All regions connected to } \\
\text { Posterior CC }\end{array}$ & $0.234(0.076)$ & $\begin{array}{l}0.401 \\
(0.024) *\end{array}$ & $\begin{array}{l}-0.227 \\
(0.394)\end{array}$ & $\begin{array}{l}-0.247 \\
(0.162)\end{array}$ & $\begin{array}{l}0.255 \\
(0.083)\end{array}$ \\
\hline${ }^{*} p<0.05$ & & & & & \\
\hline
\end{tabular}


Table 3

Correlations between SHIC and clinical disability

\begin{tabular}{|c|c|c|c|c|c|c|}
\hline $\begin{array}{l}\text { Mean sHIC extracted from } \\
\text { cortical regions }\end{array}$ & BVMT & PASAT & SDMT & $\begin{array}{l}\text { 25-Foot } \\
\text { Walk }\end{array}$ & 9-HPT & EDSS \\
\hline Cuneus & $\begin{array}{l}0.468 \\
(0.016) *\end{array}$ & $\begin{array}{l}0.318 \\
(0.168)\end{array}$ & $\begin{array}{l}0.423 \\
(0.009) *\end{array}$ & $\begin{array}{l}0.399 \\
(0.029) *\end{array}$ & $\begin{array}{l}0.295 \\
(0.188)\end{array}$ & $\begin{array}{l}-0.372 \\
(0.074)\end{array}$ \\
\hline Lateral occipital & $\begin{array}{l}0.457 \\
(0.009) *\end{array}$ & $\begin{array}{l}0.407 \\
(0.051)\end{array}$ & $\begin{array}{l}0.343 \\
(0.049) *\end{array}$ & $\begin{array}{l}0.341 \\
(0.048) *\end{array}$ & $\begin{array}{l}0.366 \\
(0.031) *\end{array}$ & $\begin{array}{l}-0.267 \\
(0.201)\end{array}$ \\
\hline Lingual & $\begin{array}{l}0.487 \\
(0.018) *\end{array}$ & $\begin{array}{l}0.264 \\
(0.217)\end{array}$ & $\begin{array}{l}0.215 \\
(0.165)\end{array}$ & $\begin{array}{l}0.343 \\
(0.104)\end{array}$ & $\begin{array}{l}0.244 \\
(0.424)\end{array}$ & $\begin{array}{l}-0.367 \\
(0.105)\end{array}$ \\
\hline Paracentral & $\begin{array}{l}0.367 \\
(0.093)\end{array}$ & $\begin{array}{l}0.431 \\
(0.018) *\end{array}$ & $\begin{array}{l}0.367 \\
(0.024) *\end{array}$ & $\begin{array}{l}0.253 \\
(0.234)\end{array}$ & $\begin{array}{l}0.224 \\
(0.343)\end{array}$ & $\begin{array}{l}-0.349 \\
(0.100)\end{array}$ \\
\hline Parstriangularis & $\begin{array}{l}0.384 \\
(0.205)\end{array}$ & $\begin{array}{l}0.449 \\
(0.040)\end{array}$ & $\begin{array}{l}0.268 \\
(0.087)\end{array}$ & $\begin{array}{l}0.139 \\
(0.812)\end{array}$ & $\begin{array}{l}0.315 \\
(0.217)\end{array}$ & $\begin{array}{l}-0.156 \\
(0.974)\end{array}$ \\
\hline Pericalcarine & $\begin{array}{l}0.497 \\
(0.011) *\end{array}$ & $\begin{array}{l}0.297 \\
(0.146)\end{array}$ & $\begin{array}{l}0.290 \\
(0.068)\end{array}$ & $\begin{array}{l}0.486 \\
(0.008) *\end{array}$ & $\begin{array}{l}0.434 \\
(0.037) *\end{array}$ & $\begin{array}{l}-0.537 \\
(0.004) *\end{array}$ \\
\hline Postcentral & $\begin{array}{l}0.388 \\
(0.035) *\end{array}$ & $\begin{array}{l}0.386 \\
(0.014) *\end{array}$ & $\begin{array}{l}0.380 \\
(0.019) *\end{array}$ & $\begin{array}{l}0.229 \\
(0.269)\end{array}$ & $\begin{array}{l}0.202 \\
(0.407)\end{array}$ & $\begin{array}{l}-0.360 \\
(0.058)\end{array}$ \\
\hline Posterior cingulate & $\begin{array}{l}0.071 \\
(0.454)\end{array}$ & $\begin{array}{l}0.345 \\
(0.026) *\end{array}$ & $\begin{array}{l}0.311 \\
(0.088)\end{array}$ & $\begin{array}{l}0.075 \\
(0.489)\end{array}$ & $\begin{array}{l}0.028 \\
(0.629)\end{array}$ & $\begin{array}{l}-0.107 \\
(0.379)\end{array}$ \\
\hline Precentral & $\begin{array}{l}0.381 \\
(0.068)\end{array}$ & $\begin{array}{l}0.278 \\
(0.122)\end{array}$ & $\begin{array}{l}0.356 \\
(0.025)^{*}\end{array}$ & $\begin{array}{l}0.281 \\
(0.190)\end{array}$ & $\begin{array}{l}0.127 \\
(0.808)\end{array}$ & $\begin{array}{l}-0.398 \\
(0.046) *\end{array}$ \\
\hline Superior parietal & $\begin{array}{l}0.255 \\
(0.094)\end{array}$ & $\begin{array}{l}0.390 \\
(0.047) *\end{array}$ & $\begin{array}{l}0.489 \\
(0.005) *\end{array}$ & $\begin{array}{l}0.281 \\
(0.056)\end{array}$ & $\begin{array}{l}0.159 \\
(0.233)\end{array}$ & $\begin{array}{l}-0.289 \\
(0.067)\end{array}$ \\
\hline Transverse temporal & $\begin{array}{l}0.275 \\
(0.178)\end{array}$ & $\begin{array}{l}0.316 \\
(0.058)\end{array}$ & $\begin{array}{l}0.397 \\
(0.015) *\end{array}$ & $\begin{array}{l}0.238 \\
(0.233)\end{array}$ & $\begin{array}{l}0.113 \\
(0.691)\end{array}$ & $\begin{array}{l}-0.387 \\
(0.035) *\end{array}$ \\
\hline $\begin{array}{l}\text { All regions connected to } \\
\text { Posterior CC }\end{array}$ & $\begin{array}{l}0.416 \\
(0.041) *\end{array}$ & $\begin{array}{l}0.369 \\
(0.046) *\end{array}$ & $\begin{array}{l}0.378 \\
(0.019) *\end{array}$ & $\begin{array}{l}0.309 \\
(0.126)\end{array}$ & $\begin{array}{l}0.230 \\
(0.345)\end{array}$ & $\begin{array}{l}-0.356 \\
(0.090)\end{array}$ \\
\hline${ }^{*} p<0.05$ & & & & & & \\
\hline
\end{tabular}

Figures 


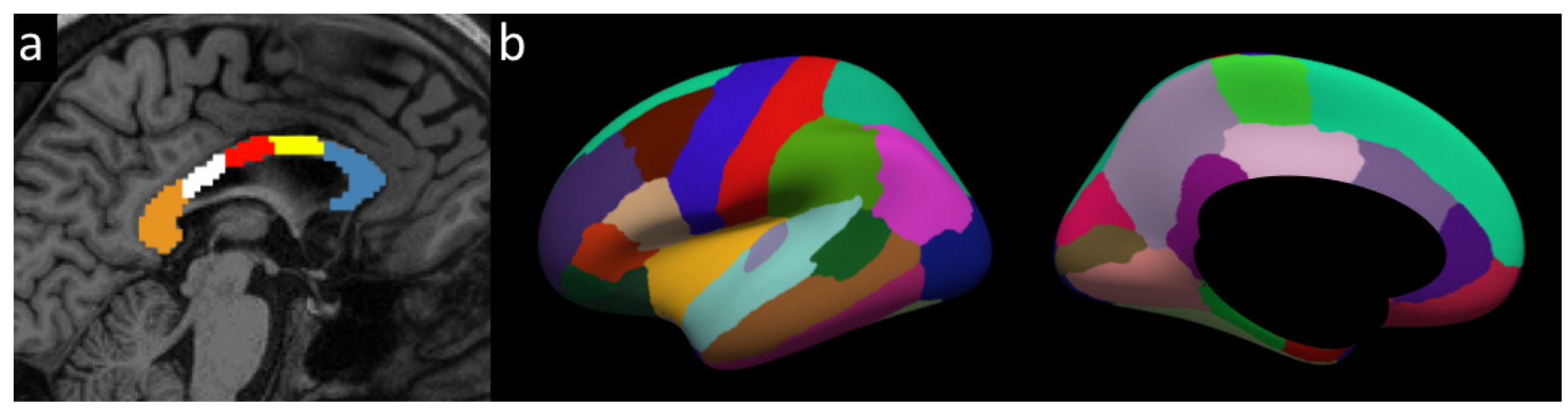

\section{Figure 1}

(a) Corpus callosum segmentation for a participant with MS (left to right: posterior, mid-posterior, central, mid-anterior, and anterior (C). Diffusion measures were extracted for each segment. (b) Desikan-Killiany atlas visualized using a left hemisphere symmetric surface template. Surface-based homologous interhemispheric connectivity (sHIC) was calculated for each cortical region. A structural connectivity matrix was generated using HCP data to define connections between the CC segments and cortical regions. 


\section{$\mathrm{HC}$}
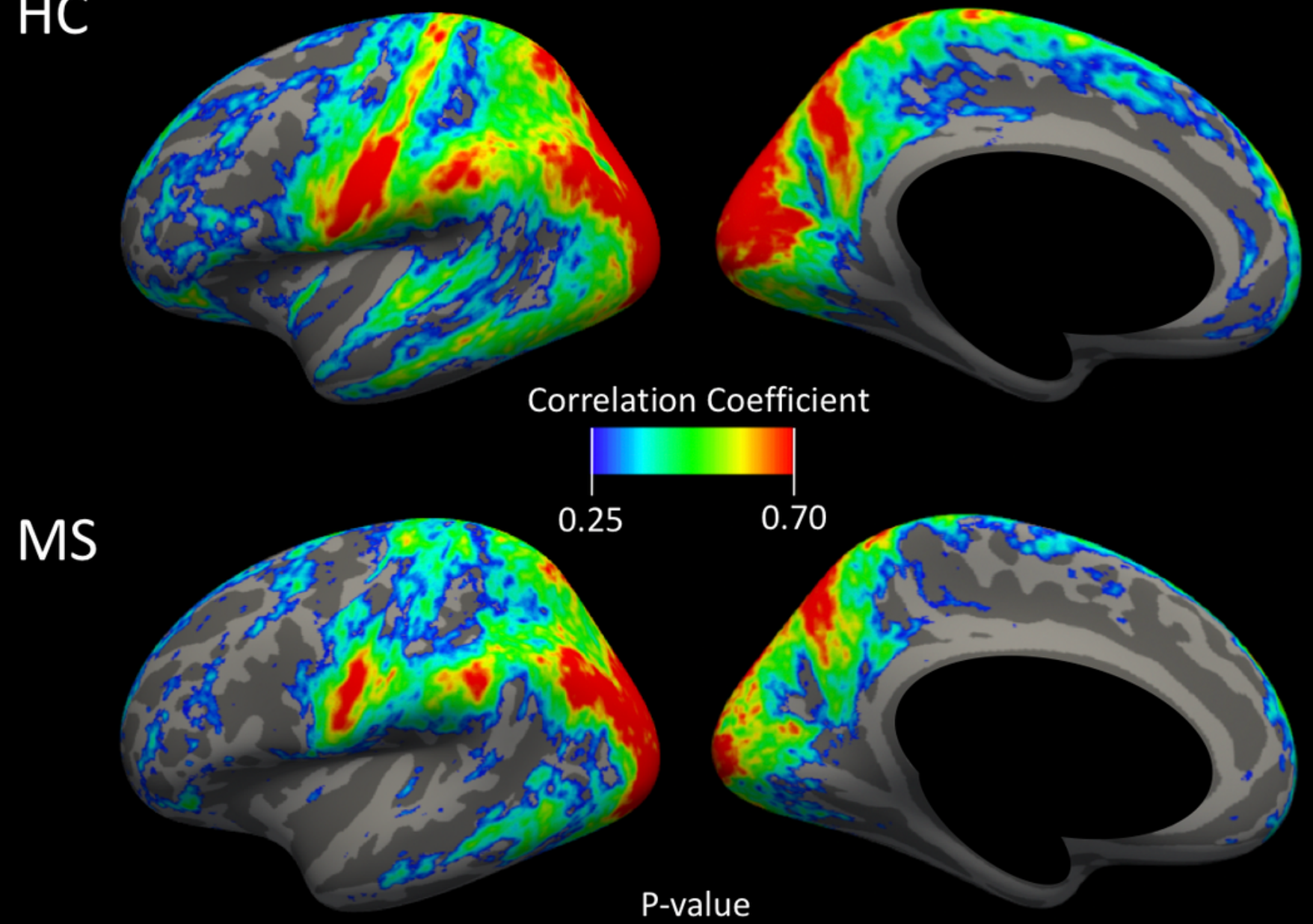

MS vs HC

\section{Figure 2}

Comparison of sHIC between participants with MS and healthy controls. Average sHIC correlation maps are shown for the MS and HC groups. Significant differences in the MS vs HC groups were detected using a vertex-wise general linear model controlling for age and gender (cluster-level $p$-values $<0.05$ ). Blue clusters represent cortical areas where sHIC was significantly reduced in the MS group. There were no areas displaying higher SHIC in the MS group 

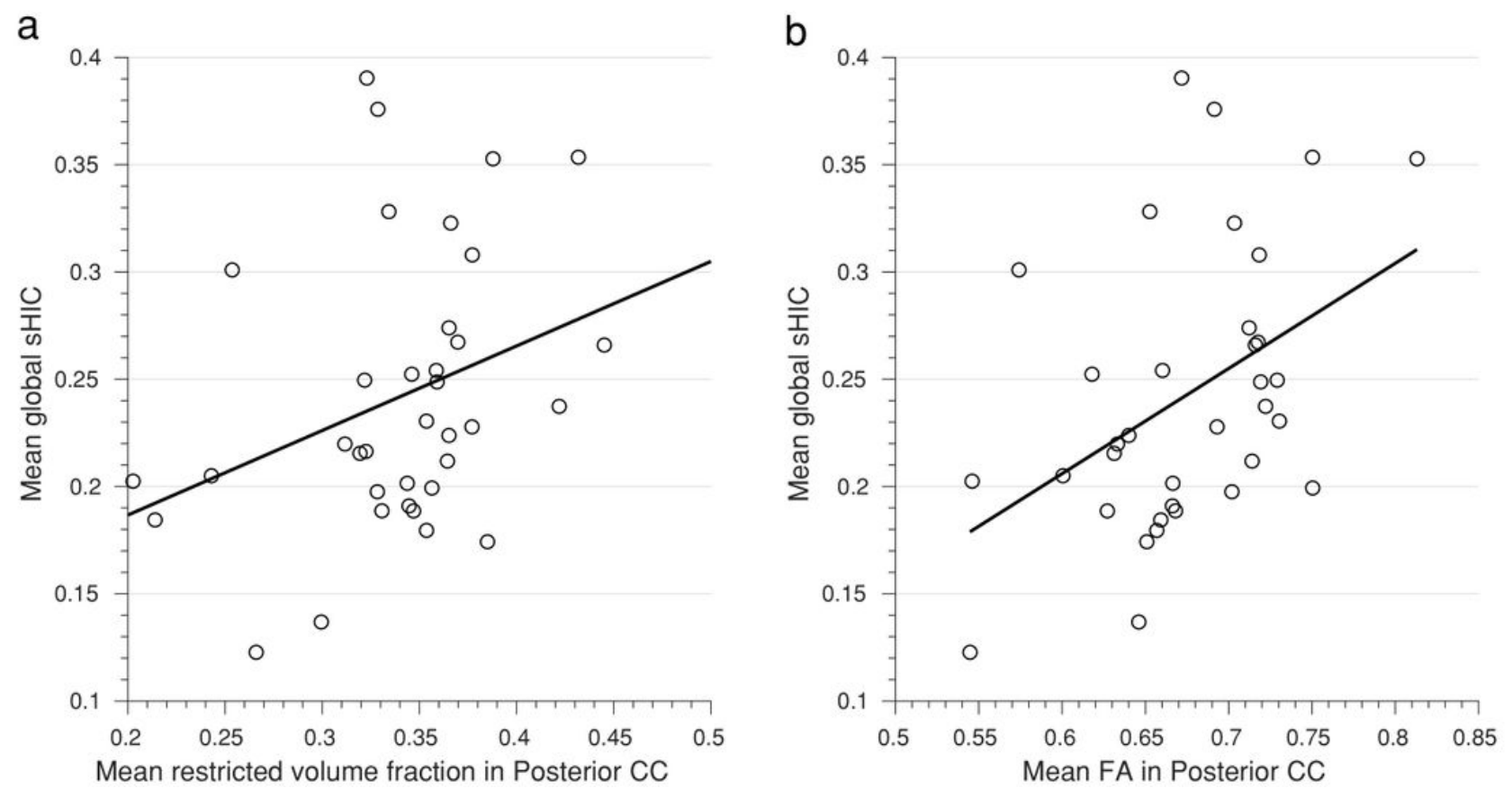

\section{Figure 3}

In participants with MS, lower global sHIC correlated with microstructural alterations in the posterior CC as measured by (a) restricted volume fraction $(r=0.325, p=0.021)$ and $(b) F A(r=0.433, p=0.013)$

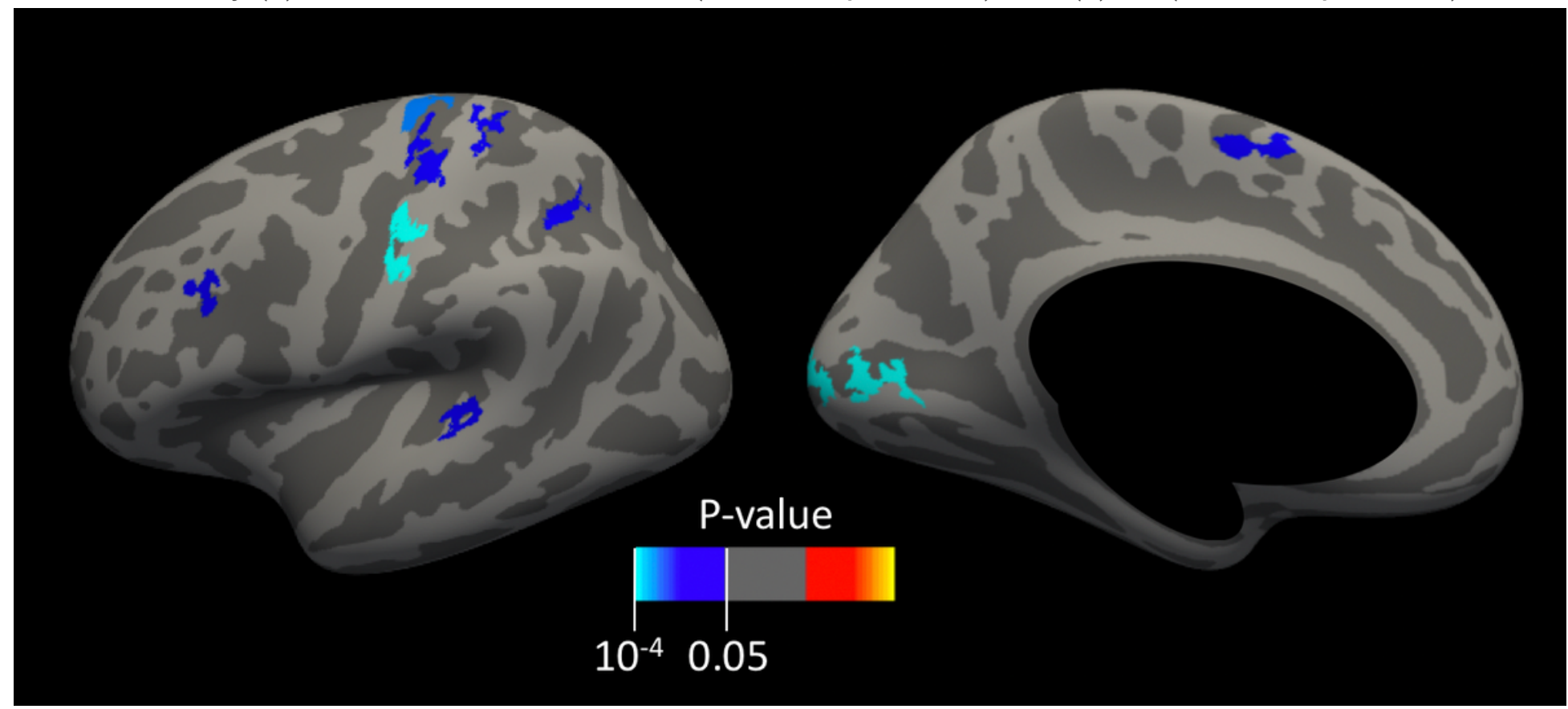

\section{Figure 4}

Associations between SHIC and EDSS in participants with MS. Blue clusters represent cortical areas where there is a significant negative association between SHIC and EDSS. Clusters were generated from a vertex-wise general linear model controlling for age and gender (cluster-level p-values < 0.05 ) 

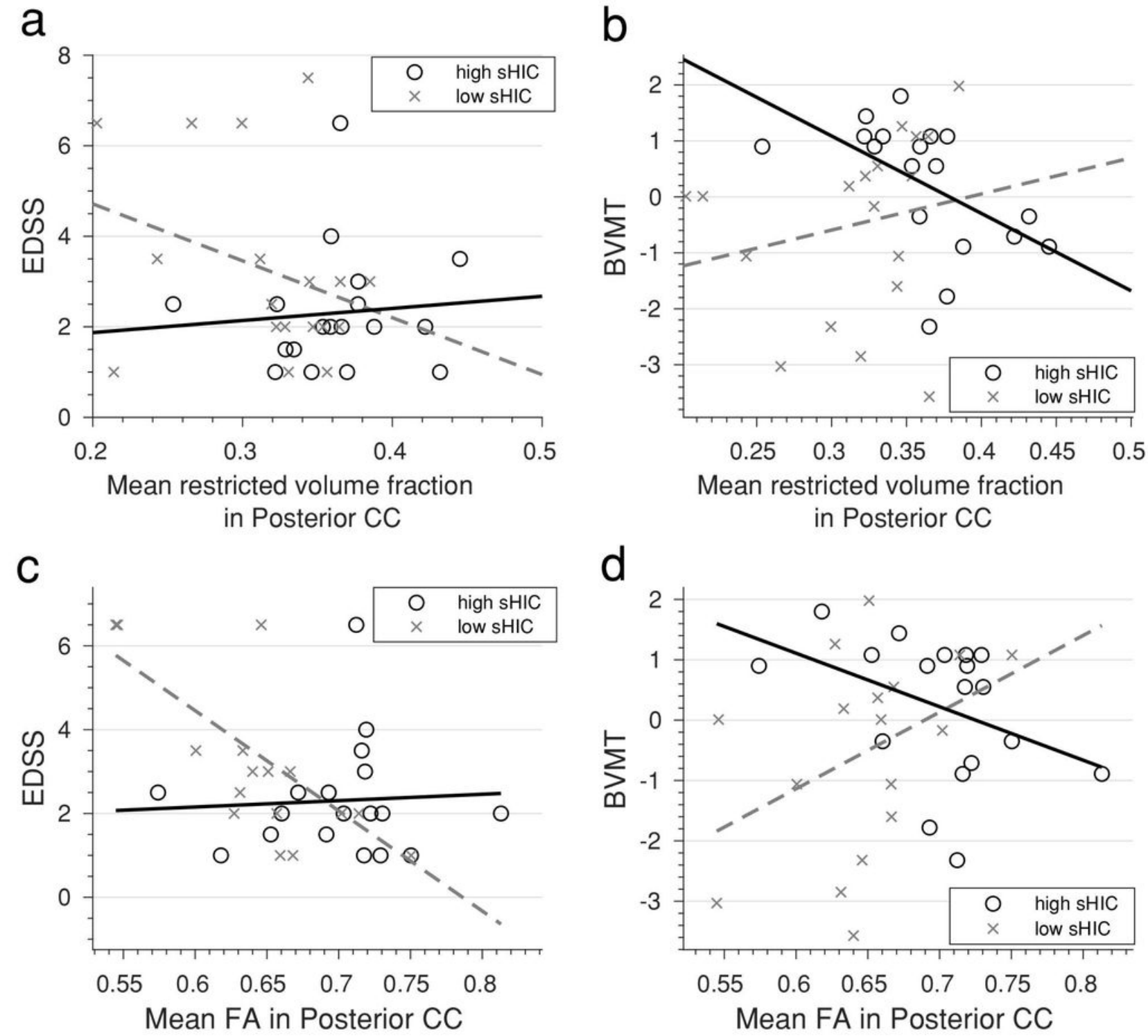

Figure 5

Interactions between SHIC, CC damage, and disability. For visualization, participants with MS were divided equally into two groups (low and high SHIC) based on the median group value for posterior sHIC. For the group with low posterior SHIC there was a significant positive correlation between posterior CC damage, measured by restricted volume fraction, and clinical disability and cognitive dysfunction as measured by (a) EDSS and (b) BVMT. This positive correlation was attenuated for the group with higher posterior SHIC. This phenomenon was also observed for FA in the posterior $\mathrm{CC}$ and the clinical measures (c) EDSS and (d) BVMT 